\title{
RELAÇÃO ENTRE ÓBITOS DE MULHERES POR AGRESSÃO, VIOLÊNCIA E ASPECTOS SOCIOECONÔMICOS NOS MUNICÍPIOS BRASILEIROS
}

Relationship between women's deaths by aggression, violence, and socioeconomic aspects in Brazilian municipalities

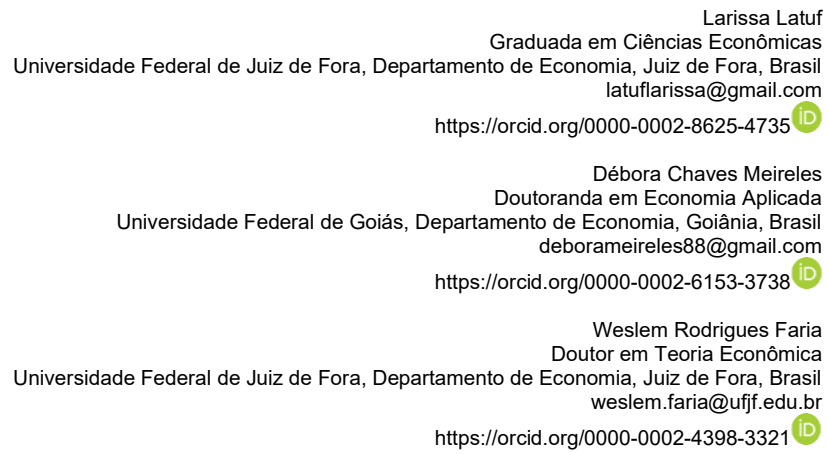

\section{RESUMO}

Objetivo: analisar a correlação entre dois conjuntos de variáveis. Um deles é formado pelo número de óbitos de mulheres por agressão e violência doméstica, sexual e outras violências. O outro é formado por indicadores socioeconômicos como o número de mulheres mães e chefes de famílias, pobreza, taxa de fecundidade, analfabetismo, renda per capita, número de empregados mulheres e salários recebidos por mulheres. Método: o método de correlação canônica foi aplicado. Resultado: os principais resultados indicaram dois coeficientes de correlação significativos entre os dois pares de variáveis canônicas geradas. Conclusões: municípios que apresentaram alto número de óbitos de mulheres por agressão e menor número de registros de violência doméstica, sexual e outras violências detinham também maior número de mulheres chefe de famílias e com filhos menores de 15 anos, maior percentual de mães jovens, maior número de empregados homens e mulheres, mas as mulheres recebiam menores salários e a proporção de pobres era maior.

PALAVRAS-CHAVE: Óbitos de mulheres por agressão. Violência doméstica e sexual. Aspectos socioeconômicos. Análise de correlações canônicas.

\begin{abstract}
Objective: to analyze the correlation between two sets of variables. One of them is formed by the number of deaths of women due to aggression and domestic, sexual and other violence. The other is formed by socioeconomic indicators such as the number of women mothers and heads of families, poverty, fertility rate, illiteracy, per capita income, number of female employees and wages received by women. Methods: The canonical correlation method was applied. Results: the main results indicated two correlation coefficients significant between the two pairs of canonical variables generated. Conclusions: municipalities that had a high number of women's deaths due to aggression and a lower number of records of domestic, sexual and other violence also had a higher number of female heads of households with children under 15 years of age, a higher percentage of young mothers, a higher number of men and women were employed, but women received lower salaries and the proportion of poor was higher.

KEYWORDS: Deaths of women due to aggression. Domestic and sexual violence. Socioeconomic aspects. Analysis of canonical correlations.
\end{abstract}

Classificação JEL: J12, J16, J31

Recebido em: 18-01-2021. Aceito em: 18-01-2022. 


\section{INTRODUÇÃO}

No mundo, 35\% das mulheres foram vítimas de violência física e/ou sexual e possuem em maior parte os seus parceiros como agressores, refletindo a subordinação, poder e controle dos homens contra as mulheres que pode chegar ao ápice da violência com o ato do feminicídio - homicídios contra as mulheres -, provocados em razão da discriminação à condição feminina (OMS, 2013). Em 2013, o Brasil ocupou o quinto lugar no ranking mundial de feminicídio com 4,8 assassinatos para cada 100 mil mulheres (ONU Mulheres, 2016). No período entre 1980 e 2013, mais de cem mil pessoas chegaram ao óbito, apenas pela condição de serem mulheres, e, principalmente, as negras e com menor nível de escolaridade (MAPA DE VIOLÊNCIA, 2015).

Há uma crescente literatura que investiga a violência doméstica no mundo. Aizer (2010) mostrou que o aumento no poder de barganha de mulheres dentro do domicílio está associado com a redução de 10\% da violência doméstica na Califórnia, entre 1990 e 2003. Resultados semelhantes foram observados por Farmer e Tiefenthaler (1996) e Gelles (1976). Esses trabalhos indicaram que a independência financeira da mulher aumenta a probabilidade de deixar o parceiro, o que leva o fim do relacionamento abusivo e da violência. Com exceção de Lenze e Klasen (2017), que mostraram que na medida em que os salários das mulheres aumentam, os homens sentem-se ameaçados em seu papel tradicional de gênero e, portanto, a violência contra as mulheres aumenta.

No Brasil, os Decretos n 1.973, de 01 de agosto de 1996, e $n^{\circ} 4.377$, de 13 de setembro de 2002, regulamentaram a Lei $\mathrm{n}^{\circ}$ 11.340, de 07 de agosto de 2006, conhecida como Lei Maria da Penha, que criou mecanismos legais para coibir a violência doméstica e familiar contra a mulher ${ }^{1}$ (BRASIL, 2006). A implementação da Lei foi um passo primordial de medidas tomadas pelas autoridades para combater o problema da violência doméstica e apresentou-se como um processo de desnaturalização da violência como parte do convívio familiar e, também, para o empoderamento das mulheres. Ainda, segundo a SPM (2012), o número de denúncias aumentou em 600\% desde a promulgação, retratando que as mulheres começaram a ter maior reconhecimento sobre seus direitos.

\footnotetext{
1 A violência doméstica refere-se a agressões, baseadas no gênero, ocasionadas por uma relação de convivência íntima ou familiar entre o agressor e a vítima, não sendo necessariamente ocorrida dentro da residência domiciliar. Além disso, são consideradas como formas de violência doméstica e familiar as seguintes classificações: violência patrimonial, sexual, física, moral e psicológica.
} 
Os estudos nacionais de Cerqueira et al. (2015), Perova e Reynolds (2017) e Schiavon (2017) utilizaram métodos experimentais para analisar a efetividade de políticas públicas implementadas no país no combate da violência contra a mulher. Garcia et al. (2016) e Martins (2017) buscaram evidenciar os determinantes da violência doméstica no Brasil associando episódios que podem favorecer a ocorrência do crime. Enquanto os estudos de Meneghel et al. (2017) e Barufaldi et al. (2017), analisaram a ocorrência da mortalidade feminina por agressão associada com fatores socioeconômicos dos municípios brasileiros.

A contribuição deste estudo é apresentar a correlação, e a forma que esta se manifesta entre dois conjuntos de variáveis, para os municípios brasileiros no ano de 2010. Um grupo é formado pelo indicador de óbitos por agressão de mulheres e pelo indicador de violência doméstica, sexual e outras violências. O outro grupo é formado por aspectos socioeconômicos, que compreendem indicadores de habitação, demografia, renda e vulnerabilidade. Para isso, foi utilizada a técnica estatística multivariada de análise de correlações canônicas. Basicamente, essa técnica testa a significância estatística da correlação existente entre dois conjuntos de variáveis, via correlação obtida entre as variáveis canônicas geradas, bem como apresenta as cargas canônicas que indicam as combinações lineares entre cada variável original e a variável canônica correspondente. Os dados sobre o número de óbitos de mulheres por agressão foram obtidos do Sistema de Informações sobre Mortalidade (SIM). Os dados sobre o número de registros de violência doméstica, sexual e outras violências foram extraídos do Sistema de Informação de Agravos de Notificação (SINAN). Os dados dos indicadores socioeconômicos foram obtidos no Atlas de Desenvolvimento Humano no Brasil e RAIS.

Os principais resultados indicaram dois coeficientes de correlação significativos entre os dois pares de variáveis canônicas geradas. As associações relacionadas à correlação mais alta, correspondente ao primeiro par de variáveis canônicas, indicaram que municípios que apresentaram alto número de óbitos de mulheres por agressão e menor número de registros de violência doméstica, sexual e outras violências eram aqueles que também detinham: um maior número de mulheres chefes de famílias e com filhos menores de 15 anos; uma maior \% de mulheres de 10 a 17 anos que tiveram filhos; possuíam maior número de empregados homens e mulheres, mas as mulheres recebiam menores salários, em média, e a proporção de pobres era maior.

Além desta introdução, o artigo conta com mais quatro seções. A segunda está destinada às discussões teóricas e às evidências empíricas sobre a violência doméstica 
contra a mulher. A terceira seção descreve os dados e o método empregado. Na quarta seção são apresentadas as análises dos resultados. E, por fim, a quinta seção tece as considerações finais deste estudo.

\section{FATORES RELACIONADOS À VIOLÊNCIA DOMÉSTICA NO BRASIL: UMA REVISÃO SOBRE A LITERATURA RECENTE}

Apesar das mudanças nas condições econômicas das mulheres ajudarem a reduzir a violência doméstica, é importante que também sejam acompanhadas por mudanças nas leis. A mudança mais evidente foi o aumento da punição dos agressores nesse tipo de crime. Contudo, isso pode ter efeitos inesperados. Por exemplo, a imputação de uma maior pena para os crimes por agressão contra as mulheres pode diminuir o incentivo destas denunciarem seus agressores, em casos em que são mais vulneráveis ou dependem financeiramente dos homens.

No Brasil, em 07 de agosto de 2006, foi promulgada a Lei $n^{0} 11.340$, conhecida popularmente como a Lei Maria da Penha, considerada o principal marco no enfrentamento da violência doméstica e familiar contra as mulheres no país, alterando os instrumentos para a condenação e processos contra os agressores e incluindo todas as pessoas que se identificam como do sexo feminino, sejam cisgêneras ou transgêneras. A lei configura como violência doméstica e familiar contra a mulher qualquer ato baseado no gênero que lhe cause lesão, sofrimento físico, social ou psicológico, dano moral ou patrimonial e a morte. Sendo assim, a medida contempla vítimas em situação de vulnerabilidade, seja o agressor o parceiro íntimo, pai, irmão ou qualquer um que seja do seu convívio. Além da prisão do suspeito, estão entre as cláusulas da Lei: a assistência médica e econômica à vítima, ordem de afastamento do agressor à vítima e a impossibilidade de substituir a pena através de multas e doações de cesta básica (BRASIL, 2006).

A Lei $n^{\circ} 13.104$, de 9 de março de 2015, conhecida como a Lei do Feminicídio, foi promulgada alterando o Código Penal, para que o feminicídio seja previsto como circunstância qualificadora do crime de homicídio. A Lei $n^{\circ}$ 8.072, de 25 de julho de 1990, caracteriza o feminicídio como crime hediondo. A pena prevista é de 12 a 30 anos e a lei prevê o aumento em um terço em ocorrência de crime nos seguintes casos: contra menor de 14 anos, maior de 60 ou pessoa com deficiência, contra gestantes ou nos três meses posteriores ao parto ou na presença de descendentes ou ascendentes da vítima (BRASIL, 2015). 
Os estudos do Cerqueira et al. (2015) e de Schiavon (2017) utilizaram as informações sobre taxa de mortalidade retiradas do SIM, para analisar a efetividade da Lei Maria da Penha, a partir do método de diferenças em diferenças. Cerqueira et al. (2015) mostraram que os mecanismos legais para coibir a violência doméstica contra a mulher contribuíram para uma queda de $10 \%$ na taxa de homicídios de mulheres praticados dentro da residência das vítimas, no período entre 2000 e 2011. Schiavon (2017) avaliou o efeito da Lei Maria da Penha sobre a taxa de homicídios femininos nos municípios brasileiros entre 2001 e 2014. Os resultados desse estudo indicaram que a Lei Maria da Penha impediu um aumento de $19 \%$ nessa taxa, sendo o efeito concentrado em municípios com menos de 100.000 mil habitantes. Entre 2007 e 2014, as evidências desse estudo indicaram que, nesses municípios, a lei resultou em uma queda de $38,6 \%$ na taxa de homicídios femininos domiciliares. Foi encontrado também um efeito da lei ainda maior para mulheres com menor nível de escolaridade, menores taxas de participação no mercado de trabalho, menores salários e menores taxas de divórcio.

A criação das delegacias de defesa das mulheres (DEAMs) foi outra política implementada no Brasil para o combate da violência contra a mulher. Foi a partir de 2003, com o lançamento da Política Nacional de Prevenção, Enfrentamento e Erradicação da Violência contra a Mulher, que o crescimento das delegacias de mulheres se desenvolveu. Perova e Reynolds (2017) estimaram o impacto das DEAMs no Brasil sobre a taxa de homicídios a nível municipal. A partir dos dados da Pesquisa Nacional por Amostra de Domicílios (PNAD) do Instituto Brasileiro de Geografia e Estatística (IBGE) e do SIM, empregaram o método de diferenças em diferenças, considerando o período de 2004 a 2009. Os resultados indicaram uma queda de $17 \%$ dos homicídios após a criação das DEAMs nas cidades brasileiras no período analisado. Os efeitos estimados foram ainda maiores para as mulheres jovens em áreas metropolitanas. Sendo assim, para tornar os DEAMs mais acessíveis e eficazes para a população em geral, sugeriu-se a instalação de outras unidades fora dos ambientes urbanos, assim como políticas e programas que facilitem a autonomia econômica das mulheres e instrumentos de capacitação e inclusão produtiva para quebrar a barreiras que naturalizam o ciclo da violência.

Garcia et al. (2016) analisaram os fatores associados ao atendimento das vítimas de violência doméstica e familiar nos serviços de urgência e emergência no Brasil. O estudo utilizou os dados do Sistema de Vigilância de Violência e Acidentes (VIVA) para o ano de 2011 e estimaram o modelo logístico não-condicional. Foi encontrado que as ocorrências de vítimas procurando atendimento estavam associadas aos finais de semana, bem como 
os períodos da noite e da madrugada. Com isso, nesses dias e horários, o contato entre a vítima e o agressor aconteceriam com maior frequência. Além disso, nesses dias e horários, há a maior possibilidade de consumo de álcool, o que tende a favorecer os episódios de violência. $O$ estudo indica, em termos de políticas de saúde, que seja realizado o rastreamento de outros serviços que a vítimas possam ter utilizado quando a violência foi sido identificada, sugerindo que muitas mulheres vítimas da violência doméstica podem não ter sido acolhidas de forma adequada nos locais anteriormente procurados.

Martins (2017) buscou identificar os determinantes da violência doméstica contra a mulher no Brasil em 2009. Esse estudo utilizou dados da Pesquisa Nacional por Amostra de Domicílios (PNAD) e do Perfil de Informações Básicas Municipais (MUNIC) e um modelo multinível logístico de dois níveis de probabilidade (individual e de ambiente social). Os resultados indicaram que o estado civil das mulheres e a existência de filhos foram as características que mais se relacionaram positivamente com a probabilidade de vitimização. O número médio de agressão contra a mulher no ano foi de 3,07 , sendo que $42,63 \%$ do total de ocorrências tiveram indivíduos da família como o agressor, $80 \%$ dos casos ocorreram na própria residência da vítima e as principais vítimas tinham entre 20 a 49 anos. Foi observada também uma maior taxa de violência contra as mulheres não brancas e de baixa escolaridade.

Meneghel et al. (2017) analisaram a mortalidade feminina por agressão através de indicadores sociodemográficos e de saúde para o Brasil entre 2003 e 2007. Os dados utilizados foram obtidos do SIM. Foi utilizado o teste de correlação de Pearson e o estimador de mínimos quadrados ordinários para verificar a relação entre a mortalidade feminina por agressão e as variáveis socioeconômicas e demográficas. As estimativas indicaram aumento dos feminicídios, principalmente de mulheres jovens, negras, pobres, solteiras e de baixa escolaridade. Além disso, verificou-se maiores taxas de mortalidade de mulheres pobres e desprivilegiadas, em territórios de maior desigualdade, onde há presença acentuada de evangélicos, pobreza e violência estrutural.

Barufaldi et al. (2017) avaliaram o perfil da mortalidade por agressão em mulheres no Brasil, no período de 2011 a 2016. Esse estudo descritivo, realizado com base nos dados do SIM e do SINAN, indicaram que as vítimas de violência apresentaram taxas de mortalidade mais elevadas para a população feminina em geral. As mulheres mais afetadas foram negras e de baixa escolaridade. As principais violências foram físicas e sexuais, praticadas, em maioria, pelos companheiros. Familiares/conhecidos/amigos também configuraram entre os principais agressores. As violências ocorriam principalmente nas 
próprias residências das vítimas. Foi identificado que as armas de fogo e objetos cortantes foram utilizadas na maior parte dos casos de violência contra a mulher.

Vale destacar ainda que, nos últimos anos, apesar das denúncias possuírem frequente aumento no Brasil, uma característica marcante da violência doméstica foi o envolvimento íntimo entre o agressor e a vítima. Mesmo em um contexto de violência, há uma tendência da mulher não procurar as autoridades, seja por se culpar da violência sofrida, por amor ao parceiro e até mesmo por temer sua integridade física e de seus filhos (BONETTI et al., 2008). A relação da vítima com o agressor, visto como seu companheiro e pai de seus filhos, pode influenciar a mulher não tomar atitude, o que gera o problema da subnotificação, conceito conhecido como a "invisibilidade da violência" (BORSOI et al., 2009). Esses casos geram certa dificuldade na precisão dos dados, visto que a notificação é tomada como denúncia, o que dificulta o avanço nas discussões e ações concernentes ao problema. Os trabalhos citados ao longo desta seção foram sintetizados e apresentados na Tabela 1.

Tabela 1 - Síntese dos trabalhos que avaliaram fatores relacionados à violência contra a mulher no Brasil

\begin{tabular}{|c|c|c|c|c|}
\hline Autores & Método & Período & Dados & Conclusões \\
\hline $\begin{array}{l}\text { Cerqueira et } \\
\text { al. (2015) }\end{array}$ & $\begin{array}{l}\text { Modelo de } \\
\text { diferenças } \\
\quad \text { em } \\
\text { diferenças }\end{array}$ & $\begin{array}{c}2000 a \\
2011\end{array}$ & SIM & $\begin{array}{l}\text { Mecanismos legais para coibir a violência } \\
\text { doméstica contra a mulher contribuíram } \\
\text { para uma queda de } 10 \% \text { na taxa de } \\
\text { homicídios de mulheres praticados dentro } \\
\text { da residência das vítimas }\end{array}$ \\
\hline $\begin{array}{c}\text { Garcia et al. } \\
(2016)\end{array}$ & $\begin{array}{l}\text { Modelo } \\
\text { logístico } \\
\text { não- } \\
\text { condicional }\end{array}$ & 2011 & $\begin{array}{l}\text { Sistema de } \\
\text { Vigilância de } \\
\text { Violência e } \\
\text { Acidentes } \\
\text { (VIVA) }\end{array}$ & $\begin{array}{c}\text { Ocorrências de vítimas procurando } \\
\text { atendimento estavam associadas aos finais } \\
\text { de semana, bem como os períodos da } \\
\text { noite e da madrugada }\end{array}$ \\
\hline $\begin{array}{l}\text { Schiavon } \\
(2017)\end{array}$ & $\begin{array}{l}\text { Modelo de } \\
\text { diferenças } \\
\text { em } \\
\text { diferenças }\end{array}$ & $\begin{array}{c}2007 \text { e } \\
2014\end{array}$ & SIM & $\begin{array}{l}\text { Lei Maria da Penha resultou em uma } \\
\text { queda de } 38,6 \% \text { na taxa de homicídios } \\
\text { femininos domiciliares }\end{array}$ \\
\hline $\begin{array}{l}\text { Perova e } \\
\text { Reynolds } \\
(2017)\end{array}$ & $\begin{array}{l}\text { Modelo de } \\
\text { diferenças } \\
\text { em } \\
\text { diferenças }\end{array}$ & $\begin{array}{c}2004 \text { a } \\
2009\end{array}$ & PNAD e SIM & $\begin{array}{c}\text { Evidências de queda de } 17 \% \text { dos } \\
\text { homicídios após a criação das delegacias } \\
\text { de defesa das mulheres (DEAMs) nas } \\
\text { cidades brasileiras }\end{array}$ \\
\hline $\begin{array}{c}\text { Martins } \\
(2017)\end{array}$ & $\begin{array}{l}\text { Modelo } \\
\text { multinível } \\
\text { logístico }\end{array}$ & 2009 & $\begin{array}{l}\text { PNAD e } \\
\text { Perfil de } \\
\text { Informações } \\
\text { Básicas } \\
\text { Municipais }\end{array}$ & $\begin{array}{l}\text { O estado civil das mulheres e a existência } \\
\text { de filhos foram as características que mais } \\
\text { se relacionaram positivamente com a } \\
\text { probabilidade de vitimização }\end{array}$ \\
\hline
\end{tabular}




\begin{tabular}{ccccc}
$\begin{array}{c}\text { Meneghel et } \\
\text { al. (2017) }\end{array}$ & MQO & $\begin{array}{c}2003 \text { e } \\
2007\end{array}$ & SIM & $\begin{array}{c}\text { Maiores taxas de mortalidade de mulheres } \\
\text { pobres e desprivilegiadas, em territórios de } \\
\text { maior desigualdade, onde há presença } \\
\text { acentuada de evangélicos, pobreza e } \\
\text { violência estrutural }\end{array}$ \\
$\begin{array}{ccccc}\text { Barufaldi et } \\
\text { al. (2017) }\end{array}$ & $\begin{array}{c}\text { Estudo } \\
\text { descritivo }\end{array}$ & $\begin{array}{c}2011 \text { a } \\
2016\end{array}$ & $\begin{array}{c}\text { As principais violências contra a mulher } \\
\text { foram físicas e sexuais, praticadas, em } \\
\text { maioria, pelos companheiros. }\end{array}$ \\
& & & SINAN & $\begin{array}{c}\text { Familiares/conhecidos/amigos também } \\
\text { configuraram entre os principais } \\
\text { agressores. }\end{array}$ \\
\hline
\end{tabular}

Fonte: Elaboração própria.

\section{METODOLOGIA}

\subsection{Dados}

A Tabela 2 apresenta os indicadores utilizados na análise. Além disso, essa tabela mostra a descrição dos mesmos e as estatísticas descritivas (média, desvio-padrão, valor mínimo e valor máximo) de cada um. Todos os dados são relativos ao ano de 2010 para 1.293 municípios brasileiros. A amostra é composta apenas pelos 1293 municípios que apresentaram pelo menos um óbito de mulheres por agressão em 2010, conforme os dados do SIM (2019). Em 2010, de acordo com os dados do SIM (2019), 1.293 municípios tiveram pelo menos um óbito de mulheres por agressão. Alguns municípios que tiveram óbitos de mulheres por agressão não apresentaram registro de violência doméstica, sexual e outras violências. Isso ocorreu em 720 municípios dos 1.293 da amostra.

Diante disso, duas possibilidades poderiam ser adotadas para o prosseguimento da análise. A primeira seria realizar a análise apenas considerando a amostra de municípios que tiveram, ao mesmo tempo, pelo menos um óbito de mulheres por agressão e um registro de violência doméstica, sexual e outras violências. A segunda seria realizá-la considerando a amostra de 1.293 municípios. As duas possibilidades foram implementadas e verificaram-se resultados praticamente idênticos em ambas. Com isso, a estimação apresentada neste trabalho considera a amostra formada pelos 1.293 municípios que tiveram pelo menos um óbito de mulheres por agressão.

Basicamente o trabalho utiliza dois tipos de dados. O primeiro conjunto de informações refere-se aos dados sobre os indicadores de mortalidade feminina por agressão e de violência doméstica, sexual e outras violências. Os dados sobre mortalidade feminina por agressão foram obtidos no SIM (2019) por meio do sistema TABNET de 
tabulação de dados do DATASUS do Ministério da Saúde. Esses dados referem-se ao número de óbitos de mulheres de 5 a 74 anos por causas evitáveis classificados nas categorias CID-10 X85 a Y09, que incluem óbitos por agressões de várias espécies. O sistema TABNET também foi utilizado para a obtenção dos dados de violência doméstica, sexual e outras violências do SINAN (2019) e esses dados são referentes ao número de notificações de violência. De acordo com essas fontes, em 2010, foram registrados 4202 óbitos femininos por agressões e 49.070 registros de violência doméstica, sexual e outras violências. $^{2}$

O segundo conjunto de informações refere-se aos indicadores socioeconômicos. $\mathrm{O}$ objetivo principal do trabalho é verificar como os indicadores do primeiro conjunto de informações se relacionam com os indicadores do segundo conjunto de informações, isto é, verificar se correlações entre mortalidade e violência sofrida por mulheres e aspectos socioeconômicos são estatisticamente significativas e como elas se dão. Isso amplia o escopo da análise realizada por d'Oliveira et al. (2009) e Vieira et al. (2011), que focaram mais na questão da atenção primária à mulher e violência sofrida por parceiros.

Dentre os indicadores socioeconômicos utilizados estão presentes aqueles relacionados à situação da mulher enquanto mãe jovem e mãe chefe de família, infraestrutura básica de moradia (habitação), aspectos populacionais e demográficos (população, taxa de fecundidade e razão de dependência), educação (taxa de analfabetismo), renda (renda per capita), pobreza (\% de pobres) e mercado de trabalho (salário de mulheres e número de empregos formais de homens e mulheres). As informações do mercado de trabalho foram obtidas na Relação Anual de Informações Sociais (RAIS, 2021) que é um serviço que atualmente é administrado pela Secretaria de Trabalho do Ministério da Economia. As informações dos demais indicadores foram obtidas no Atlas de Desenvolvimento Humano no Brasil (2013). O Atlas é uma plataforma que possibilita organizar o extrair os dados primários dos Censos Demográficos do IBGE de 1991, 2000 e 2010.

Tabela 2 - Indicadores selecionados (2010)

\footnotetext{
2 Do total de 4202 óbitos femininos por agressão foram considerados na análise 4201 registros, uma vez que para um óbito não foi identificado o município de origem. Além disso, do total de 49070 registros de violência doméstica, sexual e outras violências, foram considerados na análise 43132 registros. Alguns municípios que apresentaram registros de violência doméstica, sexual e outras violências tiveram que ser excluídos da análise por não terem apresentado pelo menos um óbito feminino por agressão.
} 
Indicadores de óbitos por agressão e de violência

\begin{tabular}{|c|c|c|c|c|c|c|c|}
\hline Indicadores & Sigla & Descrição & Fonte & Média & $D-P^{*}$ & Mínimo & Máximo \\
\hline $\begin{array}{l}\text { Óbitos de } \\
\text { mulheres }\end{array}$ & OAG & $\begin{array}{l}\text { Número de ocorrências de } \\
\text { óbitos por causas evitáveis } \\
\text { de mulheres de } 5 \text { a } 74 \text { anos } \\
\text { classificados de acordo com } \\
\text { a categoria CID-10 X85-Y09** } \\
\text { a cada } 1000 \text { habitantes }\end{array}$ & SIM & 0,07 & 0,07 & 0,00 & 0,64 \\
\hline $\begin{array}{l}\text { Violência } \\
\text { doméstica, } \\
\text { sexual e } \\
\text { outras }\end{array}$ & VDS & $\begin{array}{l}\text { Número de ocorrências de } \\
\text { violência doméstica, sexual e } \\
\text { outras violência sofridas por } \\
\text { mulheres a cada } 1000 \\
\text { habitantes } \\
\end{array}$ & SINAN & 0,19 & 0,49 & 0,00 & 5,07 \\
\hline \multicolumn{8}{|c|}{ Indicadores socioeconômicos } \\
\hline Indicadores & Sigla & Descrição & Fonte & Média & $D-P^{*}$ & Mínimo & Máximo \\
\hline Mães jovens & PMF & $\begin{array}{l}\text { \% de mulheres de } 10 \text { a } 17 \\
\text { anos que tiveram filhos } \\
\text { Número de mulheres chefe } \\
\text { de família e com filhos }\end{array}$ & Atlas & 3,20 & 1,61 & 0,00 & 12,17 \\
\hline Mulher chefe & MCF & $\begin{array}{l}\text { menores de } 15 \text { anos (em mil } \\
\text { mulheres) a cada } 1000 \\
\text { habitantes } \\
\% \text { da população que vive em }\end{array}$ & Atlas & 0,04 & 0,01 & 0,01 & 0,10 \\
\hline Habitação & PDB & $\begin{array}{l}\text { domicílios com banheiro e } \\
\text { água encanada }\end{array}$ & Atlas & 82,48 & 19,31 & 6,68 & 100,00 \\
\hline Fecundidade & TXF & Taxa de fecundidade ${ }^{* * *}$ & Atlas & 2,15 & 0,46 & 1,23 & 4,15 \\
\hline $\begin{array}{l}\text { Razão de } \\
\text { dependência }\end{array}$ & RDP & Razão de dependência**** & Atlas & 49,97 & 8,70 & 34,05 & 92,61 \\
\hline Analfabetismo & TXA & $\begin{array}{l}\text { Taxa de analfabetismo - } 18 \\
\text { anos ou mais }\end{array}$ & Atlas & 15,34 & 10,68 & 1,55 & 46,90 \\
\hline $\begin{array}{l}\text { Renda per } \\
\text { capita }\end{array}$ & RPC & $\begin{array}{l}\text { Renda per capita (em mil } \\
\text { reais) }\end{array}$ & Atlas & 0,56 & 0,28 & 0,13 & 2,00 \\
\hline Pobreza & PPB & $\%$ de pobres & Atlas & 20,85 & 16,51 & 0,19 & 70,90 \\
\hline $\begin{array}{l}\text { Salário } \\
\text { mulheres }\end{array}$ & SAM & $\begin{array}{l}\text { Rendimento médio nominal } \\
\text { recebido por mulheres (em } \\
\text { milhões de reais) }\end{array}$ & RAIS & 17,74 & 144,3 & 0,00 & $4.278,9$ \\
\hline $\begin{array}{l}\text { Emprego } \\
\text { homens }\end{array}$ & $\mathrm{MVH}$ & $\begin{array}{l}\text { Número de empregos formais } \\
\text { ocupados por homens a cada } \\
1000 \text { habitantes (em mil) }\end{array}$ & RAIS & 0,09 & 0,07 & 0,00 & 0,55 \\
\hline $\begin{array}{l}\text { Emprego } \\
\text { mulheres }\end{array}$ & MVM & $\begin{array}{l}\text { Número de empregos formais } \\
\text { ocupados por mulheres a } \\
\text { cada } 1000 \text { habitantes (em } \\
\text { mil) }\end{array}$ & RAIS & 0,06 & 0,04 & 0,00 & 0,45 \\
\hline
\end{tabular}

Fonte: Elaboração própria com base nos dados do Atlas do Desenvolvimento Humano no Brasil (2013), SIM (2019), SINAN (2019) e RAIS (2021).

Notas: Amostra = 1293. * D-P: Desvio-padrão.

** Categoria CID-10: X85 - Agressão meio droga medicamento e subst. biológicas; X86 Agressão p/meio de subst. Corrosivas; X87 - Agressão p/pesticidas; X88 - Agressão p/meio de gases e vapores; X89 - Agressão outros prod. quím. subst. Nocivas esp.; X90 - Agressão prod. quím. e subst. nocivas NE; X91 - Agressão enforcamento, estrangulamento e sufocação; X92 - 
Agressão p/meio de afogamento e submersão; X93 - Agressão disparo de arma de fogo de mão; X94 - Agressão disparo arma fogo de maior calibre; X95 - Agressão disparo outra arma de fogo ou NE; X96 - Agressão p/meio de material explosivo; X97 - Agressão p/meio de fumaça, fogo e chamas; X98 - Agressão vapor d'água gases ou objetos quentes; X99 - Agressão objeto cortante

ou penetrante; Y00 - Agressão p/meio de um objeto contundente; Y01 - Agressão p/meio projeção de um lugar elevado; Y02 - Agressão por meio de projeção ou colocação da vítima diante de um objeto em movimento; Y03 - Agressão p/meio de impacto de um veículo a motor; Y04 - Agressão p/meio de força corporal; Y05 - Agressão sexual p/meio de força física; Y06 Negligência e abandono, Y07 - Outras síndromes de maus tratos; Y08 - Agressão p/outros meios específicos, Y09 - Agressão p/meios não especificados.

*** Número médio de filhos que uma mulher deverá ter ao terminar o período reprodutivo (15 a 49 anos de idade).

**** Razão de dependência é medida pela razão entre o número de pessoas com 14 anos ou menos e de 65 anos ou mais de idade (população dependente) e o número de pessoas com idade de 15 a 64 anos (população potencialmente ativa) multiplicado por 100.

\subsection{Análise de Correlações Canônicas}

A análise foi realizada com base no método de correlação canônica, que é uma técnica de estatística multivariada proposta por Hotelling (1935). Esse método tem o intuito de identificar e quantificar a associação entre dois conjuntos de variáveis que possuem naturezas diferentes. A proposta da técnica é o desenvolvimento de combinações lineares que expressem bem as correlações entre os dois conjuntos de variáveis, de forma que a correlação entre essas combinações seja maximizada. Com isso, são determinados os pares de combinações lineares $U=a^{\prime} X$ e $V=b^{\prime} Y$ os quais possuem a maior correlação possível. Essas combinações lineares construídas são as variáveis canônicas e suas associações são denotadas correlações canônicas (JOHNSON; WICHERN, 2002; MINGOTI, 2005).

A principal justificativa para a escolha da correlação canônica é devido ao alinhamento entre o objetivo central do artigo e a capacidade analítica desse método. $O$ objetivo é verificar como um grupo de variáveis, formado por óbitos por causas evitáveis de mulheres e ocorrências de violência doméstica, sexual e outras violências sofridas por mulheres, se correlaciona com outro grupo de variáveis, formado por indicadores socioeconômicos. Portanto, o $Y$ seria um vetor de duas variáveis. Com isso, essa análise não seria possível, por exemplo, utilizando-se modelos de regressão, que faz avaliação considerando apenas uma variável dependente. Outros métodos como análise fatorial e componentes principais também não atenderiam ao objetivo do trabalho, pois gerariam índices sintéticos com correlação nula entre eles. Logo, não seria possível avaliar a correlação entre dois grupos de variáveis utilizando-se esses métodos. 
O modelo teórico é concebido com a suposição de dois vetores aleatórios, $X$ e $Y$, de dimensões $p \times l$ e $q \times l$, respectivamente. Os vetores de médias e matrizes de covariância dos vetores $X$ e $Y$ são descritos por (MINGOTI, 2005):

$$
\begin{aligned}
& E(X)=\mu_{x} \quad E(Y)=\mu_{y} \\
& \operatorname{Var}(X)=\Sigma_{X X} \operatorname{Var}(Y)=\Sigma_{Y Y} \\
& \operatorname{Cov}(X, Y)=\Sigma_{X Y} \operatorname{Cov}(Y, X)=\Sigma_{Y X}=\Sigma_{X Y}^{\prime}
\end{aligned}
$$

As associações presentes entre as variáveis aleatórias dos vetores $X$ e $Y$ estão nas matrizes $\Sigma_{X X}$ e $\Sigma_{Y Y}$. A matriz $\Sigma_{X Y}$ mede as covariâncias entre os pares de variáveis $X$ e $Y$. $\mathrm{Na}$ medida em que o procedimento alcança uma nova etapa, são construídas duas combinações lineares, uma relativa às variáveis que estão no vetor $X$ e outra relativa às variáveis que estão no vetor $Y$ (MINGOTI, 2005).

Essas novas variáveis são as chamadas variáveis canônicas. De uma forma geral, o $k$ - ésimo par de variáveis canônicas é definido como sendo o par que contém as combinações lineares $U_{k}$ e $V_{k}$, como:

$U_{k}=a_{k}^{\prime} X \mathrm{e} V_{k}=b_{k}^{\prime} Y$

$\mathrm{Na}$ equação (4), $a_{k}$ e $b_{k}$ são vetores de constantes de dimensões $p \times l$ e $q \times l$, respectivamente. Estes são escolhidos de maneira que a correlação entre as variáveis $U_{k}$ e $V_{k}$ seja maximizada no conjunto das combinações lineares de $X$ e $Y$ e, de tal modo, que essas novas duas variáveis tenham variância iguais a 1 :

$\operatorname{Var}\left(U_{k}\right)=\operatorname{Var}\left(V_{k}\right)=1$

A correlação entre as variáveis $U_{k}$ eV $V_{k}$ é chamada de correlação canônica, sendo $k=1,2, \ldots, \min (p, q)$. A técnica de correlação canônica garante que as variáveis canônicas de um par sejam não correlacionadas com as variáveis canônicas de outro par. Os vetores $a_{k}$ e $b_{k}, \operatorname{com} k=1,2, \ldots, \min (p, q)$, são solução do seguinte sistema de equações:

$\left\{\begin{array}{l}\left(\Sigma_{X Y} \Sigma_{Y Y}^{-1} \Sigma_{Y X}-\lambda_{k} \Sigma_{X X}\right) a_{k}=0 \\ \left(\Sigma_{Y X} \Sigma_{X X}^{-1} \Sigma_{X Y}-\lambda_{k} \Sigma_{Y Y}\right) b_{k}=0\end{array}\right.$

No sistema (6), $\lambda_{k}$ satisfaz as seguintes equações características:

$\left\{\begin{array}{l}\left|\Sigma_{X Y} \Sigma_{Y Y}^{-1} \Sigma_{Y X}-\lambda_{k} \Sigma_{X X}\right|=0 \\ \left|\Sigma_{Y X} \Sigma_{X X}^{-1} \Sigma_{X Y}-\lambda_{k} \Sigma_{Y Y}\right|=0\end{array}\right.$

Assim, $\lambda_{k}$ é o k-ésimo maior autovalor da matriz $\left(\Sigma_{X X}^{-1} \Sigma_{X Y} \Sigma_{Y X}^{-1} \Sigma_{Y X}\right)$ ou, equivalentemente, da matriz $\Sigma_{Y Y}^{-1} \Sigma_{Y X} \Sigma_{X X}^{-1} \Sigma_{X Y}$. A correlação canônica é a correlação em valor absoluto entre $U_{k}$ e $V_{k}$ e é igual a $\sqrt{\lambda_{k}}$ :

$\rho_{k}^{* 2}=\lambda_{k}=\left(\operatorname{corr}\left(U_{k}, V_{k}\right)\right)^{2}=\frac{\left(a_{k}^{\prime} \Sigma_{X Y} b_{k}\right)^{2}}{\left(a_{k}^{\prime} \Sigma_{X X} a_{k}\right)\left(b_{k}^{\prime} \Sigma_{Y Y} b_{k}\right)}$ 
É importante observar que se os vetores $X$ e $Y$ forem independentes entre si, ou não correlacionados, $a_{k}{ }^{\prime} X$ e $b_{k}{ }^{\prime} Y$ terão correlação igual a zero para qualquer escolha dos vetores $a_{k} \mathrm{e} b_{k}$. Portanto, é necessário fazer uma análise da matriz de covariâncias ou de correlações cruzadas para verificar se estas são próximas ou não da matriz nula. Para fazer tal verificação, aplica-se o seguinte teste:

$H_{0}: \sum X Y=0_{p x q} ; H_{A}: \sum X Y \neq 0_{p x q}$

Na equação (9), $0_{p x q}$ é a matriz nula. A estatística do teste é dada por:

$-2 \ln (\Lambda)=-\left(n-1-\frac{1}{2}(p+q+1)\right) \ln \left(\prod_{i-1}^{p}\left(1-\lambda_{i}\right)\right)$

em que $n$ é o tamanho da amostra.

É possível também testar a significância das correlações canônicas, isto é, testa-se se as $k$ primeiras correlações são significativas a ponto de serem importantes na caracterização dos conjuntos de dados da pesquisa:

$H_{0}^{(k)}: \rho_{1}^{* 2} \neq 0, \rho_{2}^{* 2} \neq 0, \ldots, \rho_{k}^{* 2} \neq 0, \rho_{k+1}^{* 2}=\rho_{k+2}^{* 2}=\ldots=\rho_{k}^{* 2}=0$

$H_{A}^{(k)}: \rho_{i}^{* 2} \neq 0$ para algum $i \geq k+1$

A estatística do teste é definida como:

$-2 \ln (\Lambda)=-\left(n-1-\frac{1}{2}(p+q+1)\right) \ln \left(\prod_{i=k+1}^{k}\left(1-\lambda_{i}\right)\right)$

\section{ANÁLISE DOS RESULTADOS}

A Tabela 3 apresenta a matriz de correlação dos indicadores selecionados para o ano de 2010. Todos os coeficientes de correlação são estatisticamente significativos a pelo menos $10 \%$ de significância. É possível observar que o indicador de óbitos de mulheres por agressão (OAG) era positivamente correlacionado com o indicador de violência doméstica, sexual e outras violências (VDS) e também com os indicadores mulher chefes de família (MCF), renda per capita (RPC), salários recebidos por mulheres (SAM), emprego de homens (MVH) e mulheres (MVM). Os mesmos sinais foram obtidos entre o indicador de violência doméstica, sexual e outras violências (VDS) e os demais indicadores socioeconômicos.

Tabela 3 - Matriz de correlação dos indicadores selecionados 


\begin{tabular}{|c|c|c|c|c|c|c|c|c|c|c|c|c|c|c|}
\hline & & 1 & 2 & 3 & 4 & 5 & 6 & 7 & 8 & 9 & 10 & 11 & 12 & 13 \\
\hline 1 & oag & 1,00 & & & & & & & & & & & & \\
\hline 2 & vds & $-0,09$ & 1,00 & & & & & & & & & & & \\
\hline 3 & pmf & 0,07 & $-0,08$ & 1,00 & & & & & & & & & & \\
\hline 4 & mcf & $-0,20$ & 0,00 & 0,14 & 1,00 & & & & & & & & & \\
\hline 5 & pdb & $-0,05$ & 0,17 & $-0,41$ & $-0,09$ & 1,00 & & & & & & & & \\
\hline 6 & txf & 0,20 & $-0,16$ & 0,55 & 0,13 & $-0,65$ & 1,00 & & & & & & & \\
\hline 7 & $\mathrm{rdp}$ & 0,17 & $-0,18$ & 0,45 & 0,11 & $-0,82$ & 0,80 & 1,00 & & & & & & \\
\hline 8 & txa & 0,18 & $-0,22$ & 0,33 & $-0,01$ & $-0,75$ & 0,54 & 0,77 & 1,00 & & & & & \\
\hline 9 & $\mathrm{rpc}$ & $-0,21$ & 0,26 & $-0,37$ & $-0,04$ & 0,69 & $-0,61$ & $-0,77$ & $-0,80$ & 1,00 & & & & \\
\hline 10 & ppb & 0,13 & $-0,21$ & 0,38 & 0,06 & $-0,87$ & 0,66 & 0,88 & 0,89 & $-0,83$ & 1,00 & & & \\
\hline 11 & sam & $-0,07$ & 0,04 & $-0,06$ & 0,05 & 0,08 & $-0,13$ & $-0,12$ & $-0,12$ & 0,28 & $-0,11$ & 1,00 & & \\
\hline 12 & $\mathrm{mvh}$ & $-0,21$ & 0,20 & $-0,23$ & $-0,01$ & 0,54 & $-0,46$ & $-0,63$ & $-0,61$ & 0,72 & $-0,65$ & 0,20 & 1,00 & \\
\hline 13 & $\mathrm{mvm}$ & $-0,24$ & 0,22 & $-0,32$ & 0,01 & 0,53 & $-0,55$ & $-0,65$ & $-0,61$ & 0,82 & $-0,65$ & 0,27 & 0,84 & 1,00 \\
\hline
\end{tabular}

Nota: Quase todos os coeficientes de correlação são significativos a pelo menos $10 \%$ de significância.

A análise de Correlação Canônica foi utilizada para analisar a relação entre os indicadores de óbitos de mulheres por agressão e violência doméstica, sexual e outras violências e os indicadores socioeconômicos. A correlação entre esses dois conjuntos de indicadores é avaliada via a correlação entre as variáveis canônicas criadas a partir da combinação linear dos indicadores de cada grupo. O primeiro conjunto possui dois indicadores e o segundo possui doze indicadores. O número gerado de pares de variáveis canônicas considera o número de variáveis do conjunto que possui menor número de variáveis. Assim, foram gerados dois pares de variáveis canônicas. A correlação entre cada par fornece o grau de associação entre os conjuntos de indicadores. Os valores dos coeficientes encontrados são $\rho_{1}=0,419$ e $\rho_{2}=0,2023$. Testes estatísticos foram realizados para avaliar a significância desses coeficientes. Os mesmos são apresentados na Tabela 4. Os testes de Lambda de Wilks, Traço de Pillai, Traço de Lawley-Hotelling e Raiz de Roy indicaram que os dois coeficientes das correlações canônicas foram significativos a $1 \%$ de significância, quando avaliados de forma conjunta. Esse resultado indica que estatisticamente os coeficientes são diferentes de zero, simultaneamente.

Tabela 4 - Testes de significância das correlações canônicas

\begin{tabular}{lcccc}
\hline & $\begin{array}{c}\text { Lambda de } \\
\text { Wilks }\end{array}$ & $\begin{array}{c}\text { Traço de } \\
\text { Pillai }\end{array}$ & $\begin{array}{c}\text { Traço de Lawley- } \\
\text { Hotelling }\end{array}$ & $\begin{array}{c}\text { Maior raiz de } \\
\text { Roy }\end{array}$ \\
\hline Estatística & 0,791 & 0,217 & 0,256 & 0,213 \\
Estatística & 14,500 & 14,137 & 14,8627 & 24,803 \\
F & 0,000 & 0,000 & 0,000 & 0,000 \\
\hline -valor & & Fon
\end{tabular}

Fonte: Elaboração própria. 
No entanto, ainda é necessário testar se a correlação entre cada par de variáveis canônicas é significativa. A Tabela 5 apresenta os resultados do teste de Lambda de Wilks para cada coeficiente de correlação. Primeiramente, o teste é aplicado sobre todas as correlações canônicas e o resultado está presente na coluna (1-2). Em seguida, o teste estatístico é aplicado novamente, porém retirando a primeira correlação dentre as duas encontradas, ou seja, testa-se a segunda correlação canônica, cujo resultado está na coluna (2-2). De acordo com os resultados dos testes, ambas as correlações foram significativas a 1\% de significância, tanto em conjunto quanto individualmente. Assim, as duas correlações canônicas são estatisticamente significativas, o que mostra que os pares de variáveis canônicas correspondentes são adequados para representar a correlação entre as características dos dois conjuntos de variáveis aleatórios (i.e., óbitos de mulheres por agressão e violência e indicadores socioeconômicos).

Tabela 5 - Testes de significância (Lamba de Wilks) das correlações canônicas

\begin{tabular}{ccc}
\hline & $\mathbf{1 - 2}$ & $\mathbf{2 - 2}$ \\
\hline Estatística & 0,791 & 0,959 \\
Estatística F & 14,500 & 5,466 \\
$p$-valor & 0,000 & 0,000 \\
\hline
\end{tabular}

Fonte: Elaboração própria.

As variáveis de ambos os conjuntos de indicadores foram padronizadas, uma vez que as combinações lineares para a formação das variáveis canônicas são influenciadas pela escala das variáveis originais. Isso ocorre em virtude de a matriz de covariâncias ser sensível à escala de um par de variáveis. Outra vantagem da padronização das variáveis é que as cargas geradas na formação das variáveis canônicas são mais equilibradas (JOHNSON; WICHERN, 2002).

A Tabela 6 apresenta as duas combinações lineares ou variáveis canônicas de cada conjunto de variáveis que foram geradas, caracterizadas como "Óbitos e violência contra as mulheres" e "Aspectos socioeconômicos". Os dois pares de variáveis canônicas gerados apresentaram coeficientes de correlação significativos, como verificado anteriormente. Os resultados indicam o comportamento de associação, baseado nos coeficientes de correlação, entre as variáveis do mesmo par para os 1.293 municípios considerados na análise. A ponderação entre os coeficientes não-padronizados e os desvios-padrão das correspondentes variáveis definem os coeficientes padronizados, que são utilizados na 
formação das combinações lineares entre as variáveis canônicas e as variáveis originais, em cada conjunto de indicadores. Os coeficientes apresentados na Tabela 6 são chamados de cargas canônicas e permitem identificar quais variáveis que mais contribuem na formação das variáveis canônicas. Quanto maior a carga canônica, maior sua importância para explicar a variável canônica.

A primeira variável canônica (V1) do grupo "Óbitos e violência contra as mulheres" é determinada principalmente pelo número de óbito de mulheres por agressão. A formação dessa variável canônica indica a tendência nos municípios em que óbitos de mulheres e violência doméstica, sexual e outras violências são negativamente correlacionados. A primeira variável canônica (U1) do grupo "Aspectos socioeconômicos" é determinada principalmente pelas variáveis: mulheres chefes de famílias, empregados homens, empregados mulheres, renda per capita e fecundidade. A formação de U1 indica que os municípios que apresentaram maior número de mulheres chefe de famílias tendem a apresentar também maior número de empregados homens e mulheres, maior renda per capita, menor taxa de fecundidade, maior proporção de mães jovens, menor taxa de analfabetismo e menores salário de mulheres. Tem-se ainda que tais municípios tendem a apresentar maior taxa de pobreza e menor \% da população que vive em domicílios com banheiro e água encanada. O coeficiente de correlação encontrado entre V1 e U1 igual a 0,419 indica esse grau de associação entre as tendências verificadas. Isso indica que municípios que apresentaram alto número de óbitos de mulheres por agressão e baixo número de violência doméstica, sexual e outras violências apresentaram também maior número de mulheres chefe de famílias e com filhos menores de 15 anos, maior \% de mulheres de 10 a 17 anos que tiveram filhos, maior número de empregados homens e mulheres, maior proporção de pobres, menor \% da população que vive em domicílios com banheiro e água encanada, as mulheres recebiam menores salários e menores taxas de fecundidade e analfabetismo.

A interpretação acerca da formação das variáveis V2 e U2 é análoga ao caso das variáveis V1 e U1. Vale destacar que as variáveis V1 e V2 são não correlacionadas, assim como as variáveis U1 e U2. Assim, a correlação entre V2 e U2 tende a captar algum padrão diferente das associações obtidas pela correlação entre V1 e U1. A correlação significativa encontrada (e igual a 0,202 ) entre V2 e U2 indica um padrão de que municípios que apresentaram maior número de óbitos de mulheres por agressão e maior número de registros de violência doméstica, sexual e outras também detinham menor número de mulheres chefe de família, maior taxa de fecundidade, maior taxa de analfabetismo, as 
mulheres tendiam a ter menores salários, assim como o número de empregados homens e mulheres, embora a renda per capita municipal fosse maior.

Tabela 6 - Coeficientes canônicos

\begin{tabular}{|c|c|c|c|}
\hline \multicolumn{4}{|c|}{ Grupo 1: Óbitos e violência contra as mulheres } \\
\hline \multirow{2}{*}{ Indicadores } & & \multicolumn{2}{|c|}{ Combinações lineares } \\
\hline & & U1 & U2 \\
\hline Óbitos de mulheres & $x 1$ & -12.510 & 7.305 \\
\hline $\begin{array}{l}\text { Violência doméstica, sexual e } \\
\text { outras }\end{array}$ & $x 2$ & 0.868 & 1.868 \\
\hline \multicolumn{4}{|c|}{ Grupo 2: Aspectos socioeconômicos } \\
\hline \multirow{2}{*}{ Indicadores } & & \multicolumn{2}{|c|}{ Combinações lineares } \\
\hline & & V1 & V2 \\
\hline Mães jovens & $\mathrm{y} 1$ & 0.049 & 0.033 \\
\hline Mulher chefe & y2 & 46.628 & -51.975 \\
\hline Habitação & y3 & -0.028 & 0.039 \\
\hline Fecundidade & y4 & -1.313 & 0.628 \\
\hline Razão de dependência & y5 & -0.009 & 0.089 \\
\hline Analfabetismo & y6 & -0.054 & 0.018 \\
\hline Renda per capita & y7 & 1.691 & 3.424 \\
\hline Pobreza & y8 & 0.035 & -0.019 \\
\hline Salário mulheres & y9 & -0.001 & -0.001 \\
\hline Emprego homens & $y 10$ & 3.555 & -0.915 \\
\hline Emprego mulheres & y11 & 1.876 & -1.905 \\
\hline
\end{tabular}

FONTE: Elaboração própria.

\section{CONSIDERAÇÕES FINAIS}

Nos últimos anos, os debates sobre a violência contra as mulheres foram se aperfeiçoando. O processo ainda é lento e gradual, no que tange ao fortalecimento das instituições e as próprias estratégias de abordagem desse fenômeno pelas entidades responsáveis, tanto em nível federal, estadual e municipal. O objetivo desse estudo foi verificar a existência de correlação e a forma com ela se dá entre os indicadores de óbitos de mulheres por agressão e violência doméstica, sexual e outras violências sofridas por mulheres e indicadores socioeconômicos. A análise foi realizada com base nos dados do SIM, SINAN, Atlas do Desenvolvimento Humano no Brasil e RAIS para o ano de 2010. O método utilizado foi a análise de Correlação Canônica. Esse método de estatística multivariada permite avaliar a correlação e a forma com ela ocorre considerando dois 
grupos de variáveis. Um desses grupos foi formado pelas variáveis do número de óbitos de mulheres por agressão e do número de registros de violência doméstica, sexual e outras violências e o outro grupo foi formado pelas variáveis socioeconômicas.

Os resultados encontrados indicaram dois coeficientes de correlação significativos entre os dois pares de variáveis canônicas geradas. Em geral, a correlação entre essas variáveis canônicas indicou que municípios que detinham maior número de óbitos de mulheres por agressão e menor número de registros de violência doméstica, sexual e outras violências contra as mulheres tendiam também a apresentar maior número de mulheres chefe de família e com filhos menores de 15 anos, maior \% de mulheres de 10 a 17 anos que tiveram filhos e maior proporção de pobres. Além disso, foi verificado que municípios que tendiam a ter maior número de óbitos de mulheres por agressão as mulheres tendiam a apresentar menores salários e maior número de empregos. Outro padrão encontrado associado ao maior número de óbitos de mulheres por agressão e maior número de registros de violência doméstica, sexual e outras violências contra as mulheres indicou o comportamento em que menos mulheres eram chefes de família e as mulheres tendiam a ter menores salários e número de empregos.

Vale destacar que os resultados estão sujeitos a falhas na geração dos dados, principalmente de óbitos e violência. Um exemplo que foi constatado neste artigo é a existência de 720 municípios em 2010 que reportaram ter tido pelo menos um óbito de mulheres por agressão e que apresentaram nenhum registro de violência doméstica, sexual e outras violências. Algumas pesquisas têm apresentado que as dificuldades no processo de notificação da violência podem estar relacionadas a fatores como: pouco preparo profissional para diagnosticar e intervir nas situações de violência, fragilidades na estrutura e organização dos serviços, falta de credibilidade na rede de proteção e receios quanto aos procedimentos legais que poderiam advir da notificação (ARPINI et al., 2008; GONÇALVES; FERREIRA, 2002; LOBATO et al., 2012).

O presente estudo contribui para a introdução de políticas públicas com estratégias de prevenção da violência contra as mulheres, através dos indicadores socioeconômicos que podem influenciar nos determinantes das ocorrências desse tipo de crime, principalmente aqueles relacionados à vulnerabilidade da mulher (situação em que as mulheres são mães de crianças e chefe de família, mães jovens e um ambiente com alta taxa de fecundidade). Além disso, reitera-se a necessidade do aprimoramento nas unidades básicas de saúde, para que forneçam melhores condições de atendimento para as vítimas e melhor forma de envolvimento dos profissionais responsáveis pela notificação. A violência 
contra a mulher, além de ser um problema de saúde pública, é uma forma de violação dos direitos humanos. Portanto, é de extrema importância medidas mais definitivas para mudar essa realidade que atinge as mulheres no mundo como um todo.

\section{REFERÊNCIAS}

AIZER, Anna. The gender wage gap and domestic violence. American Economic Review, v. 100, n. 4, p. 1847-1859, 2010.

ARPINI, Dorian Mônica; SOARES, Adriane Cristine Oss-Emer; BERTÊ, Lidiane; FORNO, Cristiano Dal. A revelação e a notificação das situações de violência contra a infância e a adolescência. Psicologia em Revista, v. 14, n. 2, p. 95-112, 2008.

ATLAS DO DESENVOLVIMENTO HUMANO NO BRASIL. Atlas PNUD. [S.I.:s.n.], 2013. Rio de Janeiro, PNUD, IPEA, Fundação João Pinheiro. Disponível em: <http://www.atlasbrasil.org.br/2013/pt/> consulta/>. Acesso em 19 nov. 2019.

BARUFALDI, Laura Augusta; SOUTO, Rayone Moreira Costa Veloso; CORREIA, Renata Sakai de Barros; MONTENEGRO, Marli de Mesquita Silva; PINTO, Isabella Vitral; SILVA, Marta Maria Alves da; LIMA, Cheila Marina de. Violência de gênero: comparação da mortalidade por agressão em mulheres com e sem notificação prévia de violência.

Ciência e Saúde Coletiva, v. 22, n. 9, p. 2929-2938, 2017.

BONETTI, Alinne; PINHEIRO, Luana; FERREIRA, Pedro. Violência contra as mulheres e direitos humanos no Brasil: uma abordagem a partir do Ligue 180. In: XVI ENCONTRO NACIONAL DE ESTUDOS POPULACIONAIS, 2008, Caxambú, Anais... Belo Horizonte: Associação Brasileira de Estudos Populacionais, 2008. p. 1-21.

BORSOI, Tatiana dos Santos; BRANDÃO, Elaine Reis; CAVALCANTI, Maria de Lourdes Tavares. Ações para o enfrentamento da violência contra a mulher em duas unidades de atenção primária à saúde no município do Rio de Janeiro. Interface: Comunicação, Saúde, Educação, v.13, n. 28, p.165-74, 2009.

BRASIL. Presidência da República. Lei n 11.340, de 7 de agosto de 2006. Cria mecanismos para coibir a violência doméstica e familiar contra a mulher e dá outras providências. Diário Oficial da União, 08 de agosto de 2006, 2006.

BRASIL. Presidência da República. Lei n 11.104, de 9 de março de 2015. Prevê o feminicídio como circunstância qualificadora do crime de homicídio. Diário Oficial da União, 09 de março de 2015, 2015.

Cerqueira, DANIEL; MATOS, Mariana; MARTINS, Ana Paula Antunes; JUNIOR, Jony Pinto. Avaliando a efetividade da Lei Maria da Penha. Texto para discussão do IPEA n. 2.048. Brasília, mar. 2015. 
d'OLIVEIRA, Ana Flávia Pires Lucas; SCHRAIBER, Lilia Blima; HANADA, Heloisa; DURAND, Júlia. Atenção integral à saúde de mulheres em situação de violência de gênero - uma alternativa para a atenção primária em saúde. Ciência e Saúde Coletiva, v. 14, n. 4, p. 1037-1050, 2009.

FARMER, Amy; TIEFENTHALER, Jill. Domestic violence: the value of services as signals. American Economic Review, v. 86, n. 2, p. 274-279, 1996.

GARCIA, Leila Posenato; DUARTE, Elisabeth Carmen; FREITAS, Lúcia Rolim Santana de; SILVA, Gabriela Drummond Marques da. Violência doméstica e familiar contra a mulher: estudo de casos e controles com vítimas atendidas em serviços de urgência e emergência. Cadernos de Saúde Pública, v. 32, n. 4, 2016.

GELLES, Richard. Abused wives: why do they stay. Journal of Marriage and Family, v. 38, n. 4, p. 659-668, 1976.

GONCALVES, Hebe Signorini; FERREIRA, Ana Lúcia. A notificação da violência intrafamiliar contra crianças e adolescentes por profissionais de saúde. Cadernos de Saúde Pública, v. 18, n. 1, p. 315-319, 2002.

JOHNSON, Richard; WICHERN, Dean. Applied Multivariate Statistical Analysis. Prentice Hall, New Jersey, 2002.

LENZE, Jana, KLASEN, Stephan. Does women's labor force participation reduce domestic violence? Evidence from Jordan. Feminist Economics, v.23, n. 1, p. 1-29, 2017.

LOBATO, Geórgia Rosa; MORAES, Claudia Leite; NASCIMENTO, Marilene Cabral do. Desafios da atenção à violência doméstica contra crianças e adolescentes no Programa Saúde da Família em cidade de médio porte do Estado do Rio de Janeiro, Brasil.

Cadernos de Saúde Pública, v. 28, n. 9, p. 1749-1758, 2012.

MARTINS, Jayne Cecília. Determinantes da violência doméstica contra a mulher no Brasil. Dissertação (Magister Scientiae), Programa de Pós-Graduação em Economia Aplicada, Universidade Federal de Viçosa, Viçosa, 2017.

MENEGHEL, Stela Nazareth; ROSA, Bruna Alexandra Rocha da; CECCON, Roger Flores; HIRAKATA, Vania Naomi; DANILEVICZ, lan Meneghel. Feminicídios: estudo em capitais e municípios brasileiros de grande porte populacional. Ciência e Saúde Coletiva, v. 22, n. 9, p. 2963-2970, 2017.

MINGOTI, Sueli Aparecida. Análise de dados através de métodos de estatística multivariada: uma abordagem aplicada. Belo Horizonte: Editora UFMG, 2005.

ONU MULHERES. Conclusões acordadas na $57^{a}$ Comissão Jurídica e Social da Mulher. ONU Mulheres, 2013.

PEROVA, Elizaveta; REYNOLDS, Sarah Anne. Women's police stations and domestic violence: evidence from Brazil. Social Science \& Medicine, v. 174, p. 188-196, 2017. 
Relação Anual de Informações Sociais. Secretaria de Trabalho. Ministério da Economia. RAIS. [S.I.:s.n.], 2021. Disponível em: <http://bi.mte.gov.br/bgcaged/>. Acesso em 03 maio 2021.

SCHIAVON, Laura Carvalho. Essays on crime and justice. Tese (Doutora em Economia), Programa de Pós-graduação em Economia, Pontifícia Universidade Católica do Rio de Janeiro, Rio de Janeiro, 2017.

Sistema de Informação de Agravos de Notificação. Ministério da Saúde/SVS. SINAN. [S.I.:s.n.], 2019. DATASUS, TABNET. Disponível em: $<$ http://tabnet.datasus.gov.br/cgi/tabcgi.exe?Sinannet/cnv/violebr.def>. Acesso em 19 nov. 2019.

Sistema de Informações sobre Mortalidade. Ministério da Saúde/SVS/CGIAE. SIM. [S.I.:s.n.], 2019. DATASUS, TABNET. 2019. Disponível em: <http://tabnet.datasus.gov.br/cgi/deftohtm.exe?sim/cnv/evitb10br.def>. Acesso em 19 nov.

VIEIRA, Elisabeth Meloni; PERDONA, Gleici da Silva Castro; SANTOS, Manoel Antônio dos. Factors associated with intimate partner physical violence among health service users. Revista de Saúde Pública, v. 45, n. 4, p. 730-737, 2011. 